\section{The difference uses of panoramic photo and CBCT evaluation of the gnathoplasty surgery outcome}

\author{
Ayu Wahyuni, ${ }^{1}$ Muliaty Yunus, ${ }^{2}$ Muhammad Ruslin ${ }^{1}$
}

CrossMark

\title{
Abstract
}

Objective: Gnathoschisis is the most common congenital craniofacial abnormality in patients with cleft lip and/or palate ( $C L$ and/or P). The panoramic radiograph is one means to establish a diagnosis and treatment plan with two-dimension photograph. The conebeam computed tomography (CBCT) is one means of supporting the maxillofacial to get a three-dimension photograph. The aim of this study was to determine the difference in the results of the use of panoramic photos and CBCT in evaluation before and after gnathoplasty surgery.

Material and Methods: This is an observational descriptive research with cross-sectional study method. All samples that meet the suitable criteria are put in this research. The data gained by collecting status card at medical record installation about photo panoramic application in gnathoschisis patients was then compare to the examination with CBCT.

Results: The result performed shows that there is a significant difference between panoramic and $\mathrm{CBCT}$ picture. The $\mathrm{CBCT}$ provide better evaluation about teeth age and segment position of jaw curve from the cleft size. While the panoramic photo provides a widely covered area to observe facial and teeth bone.

Conclusion: The $\mathrm{CBCT}$ results are more accurate in evaluating gnathoplasty surgery than panoramic photo; however, exposure to the radiation should be considered.
'Department of Oral and Maxillofacial Surgery, Faculty of Dentistry Hasanuddin University, Makassar, Indonesia

${ }^{2}$ Department of Radiology Faculty of Dentistry Hasanuddin University, Makassar, Indonesia

*Correspondence to: Muhammad Ruslin,

mruslin@unhas.ac.id

Received: 07 January 2017

Revised: 20 May 2017

Accepted: 21 June 2017

Available online: 01 August 2017

Keywords: Cone-beam computed tomography, Gnathoplasty, Panoramic photo

Cite this Article: Wahyuni A, Yunus M, Ruslin M. 2017. The difference uses of panoramic photo and CBCT evaluation of the gnathoplasty surgery outcome. Journal of Dentomaxillofacial Science 2(2): 110-113. D01:10.15562/jdmfs.v2i2.535

\section{Introduction}

The gnathoschisisis an oro-facial deformity, which is an alveolar gate, an imperfect development during the fetus formation. The epidemiology of gnathoschisis vary depending on genetical, geographical, race, sex, cultural and socio-economic factors. ${ }^{1-3}$ Gnathoschisis in operational examination is needed to check the roentgen photo. Not only does it detect gnathoschisis and its severity, but also define the exact distance of the alveolar gate that are very important to its therapeutic planning and to evaluate operational performance. ${ }^{4}$

Radiography in dentistry is divided into intra and extra oral. The extra oral radiography which is commonly conducted in dentistry: a panoramic radiography and cone-beam computed tomography (CBCT). ${ }^{5}$ The panoramic radiography is applied to observe and identify manifestation of disease in mouth as well as a tool to support the diagnosis and to evaluate the cure in two-dimensional picture. ${ }^{5}$ In the recent year, the CBCT has been commonly applied because of its capacity to reconstruct $3 \mathrm{D}$ photo of oromaxillofacial. But, the CBCT is not suitable to determine soft tissue structure. The CBCT is used for diagnostic picture of implantology and traumatology in oromaxillafacial area. ${ }^{4}$
Technological difference in result of panoramic radiography's diagnostic picture and CBCT are different. Research data reported that CBCT had shown wide variety, with specificity score (62-100\%) and accurate score (77-96\%). ${ }^{4}$ But, the panoramic radiography data cannot be found. The aim of this report is to explain about the difference of panoramic photo with CBCT in scoring gnathoplasty operational result.

\section{Material and Methods}

This is an observational descriptive research with cross-sectional study method. It was conducted

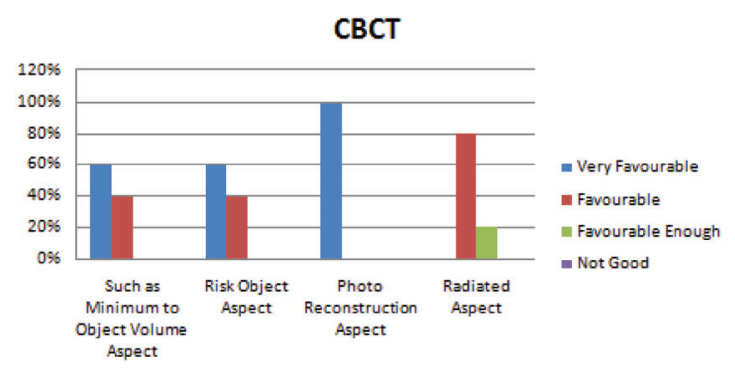

Figure 1 CBCT photo based on maximum aspect to the object volume location, risk object aspect, figure reconstructed aspect and radiated aspect 


\section{Panoramic Photo}

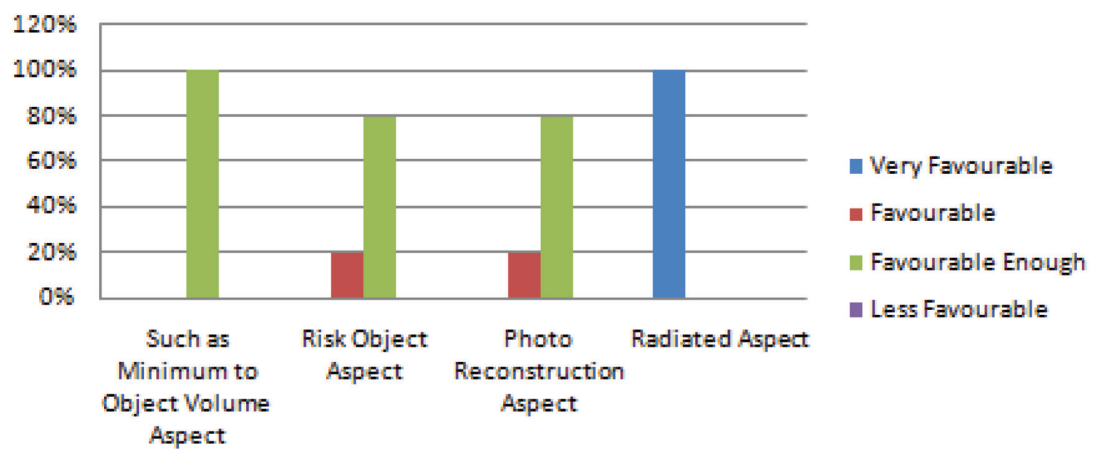

Figure 2 Panoramic photo based on maximum aspect to the object volume location, risk object aspect, figure reconstruction aspect and radiation aspect

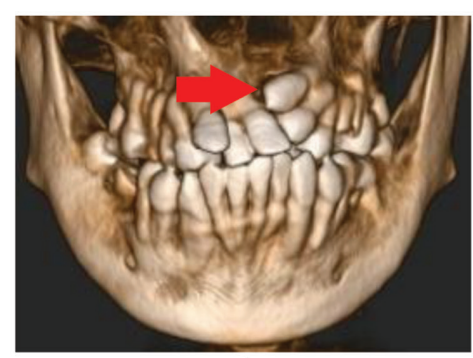

A

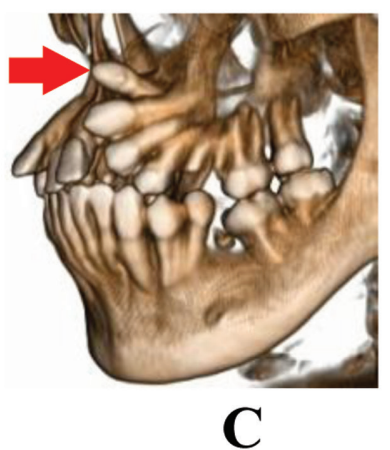

Figure $3 \mathrm{CBCT}$ evaluation result from antero-posterior A. Pre-operation, and B. Post-operation in patient with gnathoplasty technique of gnathoschisis. CBCT evaluation result from left oblique, C. Preoperation, and D. Post-operation in gnathoschisis patient with genitoplasty technique

with consecutive sampling, in which all samples that met the suitable criteria were put in this research. The research was conducted in Dental Hospital Hasanuddin University, Makassar, Indonesia from July 20,2016 to August 5, 2016 and had the ethical approval and recommendation from Hasanuddin University. Data was obtained by collecting status card at medical record installation about photo panoramic application in gnathoschisis patients, then was compare to the examination with CBCT. This research was analyzed based on 4 aspects, such as minimum to object volume aspect, risk object aspect, photo reconstruction aspect and radiated aspect.

\section{Results}

This research was carried out on samples, which were gnathoschisis patient in Dental Hospital, Hasanuddin University. The result performed showed that there is a significant difference between panoramic picture and $\mathrm{CBCT}$, which can be seen in figure 1 and 2.

Based on figure 1 the CBCT photo result indicated that maximum aspect to object volume $60 \%$ is very favorable and $40 \%$ is enough favorable, risk object aspects shows that there is $60 \%$ of very favorable result and $40 \%$ of enough favorable, figure reconstruction aspect shows that there is $100 \%$ of very favorable result, and radiation aspect shows that there is $80 \%$ of favorable result and $20 \%$ of enough favorable result. Meanwhile, in radiated aspect is enough favorable. The panoramic photo performed the result that from maximum aspect to object volume $100 \%$ is enough favorable, risk object aspect shows that there is $20 \%$ favorable result and $80 \%$ of enough favorable result, figure reconstruction aspect shows that there is $20 \%$ of favorable result and $80 \%$ of enough favorable, and radiation aspect shows that there is $100 \%$ of very favorable result. This indicates that the figure performance is satisfactory enough. Meanwhile, radiation aspect's result is very favorable figure 2 .

\section{Patient evaluation with CBCT}

Based on figure $3 \mathrm{~A}$ and $\mathrm{B}$, the $\mathrm{CBCT}$ photo result of antero-posterior shows that it performed better prediction result in morphological defect and graft material volume that needed in its improvement. The CBCT provided better evaluation about teeth age and segment position of jaw curve from the cleft size. Based on figure $3 \mathrm{C}$ and $\mathrm{D}$, the $\mathrm{CBCT}$ photo from left oblique, showed the position of teeth near cleft alveolar as well as the position of bone graft. In gnathoschisis case with gnathoplasty surgical technique the alveolar bone graft was conducted in 7-9 year-old children by considering the incidence of impaction at incisivus lateralis and caninus teeth.

\section{Patient evaluation with panoramic photo}

Based on figure 4, which is a panoramic photo result; the panoramic photo provides a wide covered area to observe facial and teeth bone. The panoramic photo can be used to observe and detect manifestation of oral cavity disease as well as a tool to support the diagnosis and to evaluate the treatment with $2 \mathrm{D}$ picture result. 

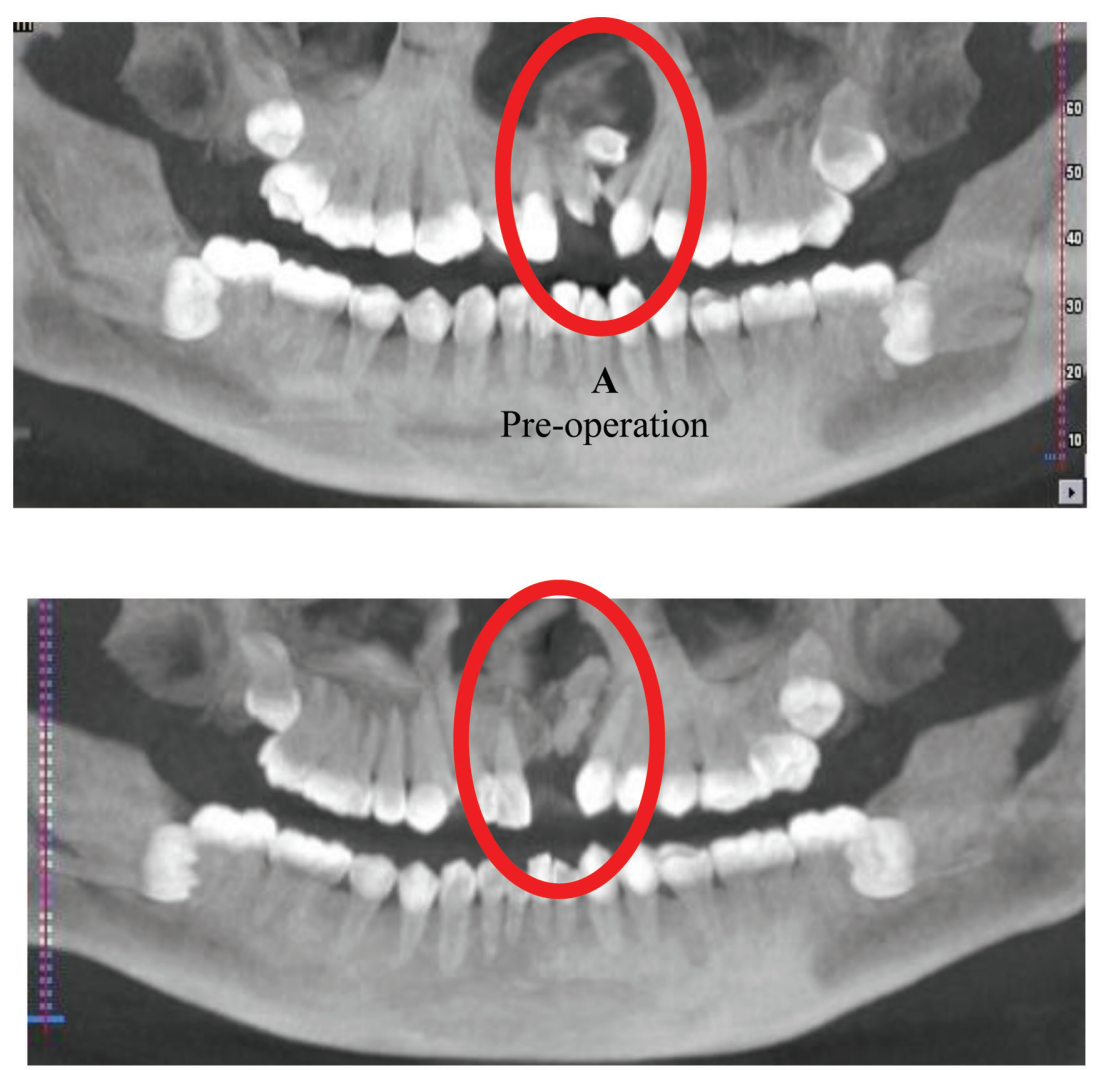

B

Post-operation

Figure 4 Evaluation result of panoramic photo A. Pre-operation and B. Postoperation in gnathoschisis patient with gnathoplasty technique

Based on figure 5 that is a panoramic photo result. Based on the case above, it has conducted an impaction at incisivus lateralis and caninus teeth, by filling cleft alveolar or leading its gap. The picture presented the result in $2 \mathrm{D}$ quality.

\section{Discussion}

This research was conducted by collecting secondary data from Dental Hospital of Hasanuddin University to determine the difference in panoramic photo application from CBCT in gnathoplasty management in gnathoschisis patient. The data collected then analyzed the comparison of its roentgen photo quality,

The alveolar bone graft was reported by Boyne and Sands in 1970's, as an important initial treatment for patient with lip and cleft palatal in achieving teeth curve, to guide permanent teeth in cleft alveolar area and to shape the alveolar bone anatomy. In the recent years, the CBCT has been applied to evaluate the treatment result of alveolar bone transplantation. But, it is complicated to apply the CBCT in daily practice because of high radiation exposure. ${ }^{6}$
According to Quereshy et al. ${ }^{7}$ in his study, he stated that treatment planning for patient with lip, alveolar, and bilateral cleft need many considerations. Because of young age of patients and worries about the radiation exposure, the conventional CT scan does not apply. The time of cleft alveolar repair is always depend on panoramic photo and occlusal radiography. Another consideration included palatal expansion as well as segmental conformity. ${ }^{7}$

The CBCT must provide better evaluation about teeth age, segment position of jaw curve of cleft size compare to traditional radiography. The volumetric analysis promises a better prediction in morphological defect and graft material volume that is needed for its restoration. A common question that appeared related to jaw curve stability after grafting, and the quality of bone graft from time to time and its effect to the face development as a whole. The CBCT provides ways to identify those issues deeply. ${ }^{7}$ This study shows material graft volume was needed in repairing alveolar gap.

In this study, based on CBCT photo as a whole that resulted in favorable picture but in high radiation level, is to be considered. Meanwhile, the panoramic photo resulted in enough favorable picture with lower radiation exposure.

Scarfe et al. in this study about CBCT in dentistry stated that recently each dentist can afford and operate a CBCT unit, which raised worries among maxillofacial radiologist based on quality and patient safety, that the CBCT application cannot be conducted by inexperienced and inadequately trained doctor. Also reminded that CBCT scan produced radiation more than maximal radiation that teeth can afford, thus to recommend maxilla Facial CBCT application doctor must go through standard training and must be conducted by competent dentist. ${ }^{6}$

In addition, Katkar et al. in her study about the CBCT application in handling cleft alveolar case stated that the CBCT provides photos to observe the position of bone graft. This study showed the width and location of alveolar bone graft. ${ }^{8}$

In gnathoschisis case with gnathoplasty surgical technique, the alveolar bone graft was conducted on 7-9-year-old children. ${ }^{9}$ In this study of cleft alveolar status, impaction has occurred, caninus teeth commonly will erupt in cleft alveolar area and erupted incisivus lateralis teeth as well as are a close to cleft alveolar will lead to those gap. Thus, gnathoplasty operation is conducted before the teeth gets erupted. The CBCT shows the position picture of teeth near cleft alveolar from left oblique.

The CBCT is able to reconstruct $3 \mathrm{D}$ photo. The superiority of CBCT make it applicable in some hospital installation. Furthermore, estimated dose 

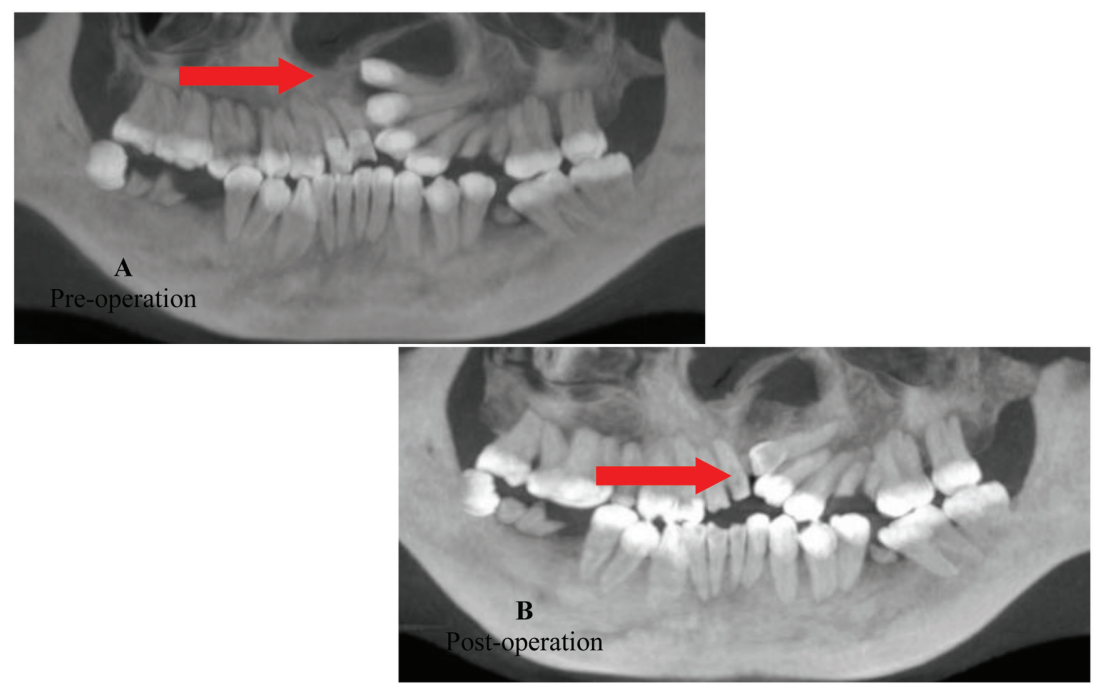

Figure 5 Panoramic photo evaluation result. A. Pre-operation and B. Postoperation in gnathoschisis patient with gnathoplasty technique

in CBCT has to be known to understand how many radiated dose can be accepted by the patient. ${ }^{10}$ However, Karkar et al. ${ }^{9}$ in his study stated that, the $\mathrm{CBCT}$ scan provides radiation in a level of more than a maximum radiation level. ${ }^{9}$

Meanwhile, the panoramic radiography has a wide coverage area to observe facial and teeth bone. One of panoramic radiography's eminence is its radiation dosage is relatively low. In this study, a panoramic photo can perform maxillomandibular in one page of film with lower radiation exposure. Meanwhile, picture result of panoramic photo s are in $2 \mathrm{D}$.

\section{Conclusion}

Gnathoplasty is an operational technique in patient with alveolar gap, therefore, to perform surgery, supported examination is needed, that is a roentgen examination to observe the condition of alveolar bone gap in more detail in 3D such as CBCT. This technique has some advantages including observable graft volume needed as well as detailed reconstructed picture. But the CBCT has some drawbacks like high-radiation dosage; when it is conducted continuously will lead to damage in candidate and erupted teeth. A panoramic photo can be applied as a roentgen examination and has a relatively low radiation dosage but result in $2 \mathrm{D}$ picture.

\section{Conflict of Interest}

The authors report no conflict of interest.

\section{References}

1. Schliephake H, Donnerstag F, Berten JL, et al. Palate morphology after unilateral and bilateral cleft lip and palate closure. Int. J. Oral Maxillofac Surg 2005;12: 13.

2. Mommaerts MY, Kablan F, Sheth S, et al. Early maxillary growth in complete cleft lip, alveolus and palate patients following Widmaier-Perko's, or a modified Furlow's technique of soft palate repair. J Craniomaxillofac Surg 2003;08: 13.

3. Ysunza A, Pamplona MC, Quiroz J, et al. Maxillary growth in patients with complete cleft lip and palate, operated on around 4-6 months of age. Int. J Pediatr Otorhinolaryngol 2010;4: 16 .

4. Linz CUDA, Mu“ller-richter, Buck AK, et al. Performance of cone beam computed tomography in comparison to conventional imaging techniques for the detection of bone invasion in oral cancer. Int J Oral maxillofac Surg 2015. p. 8-15.

5. Ahmad M, Jenny J, Downie M. Application of cone beam computed tomography in oral and maxillofacial surgery. Aust Dent J 2012;57: 82-94.

6. Scarfe WC, Farman AG, Sukovic P. Clinical applications of cone-beam computed tomography in dental practice. Fevrier2006;72: 75-80.

7. Quereshy F, Savell T, Palomo J. Applications of cone beam computed tomography in the practice of oral and maxillofacial surgery. J oral maxillofac surg 2008;66: 791-796.

8. Suomalainen A, Aberg T, Rautio Jet al. Cone beam computed tomography in the assessment of alveolar bone grafting in children with unilateral cleft lip and palate. Europ J Ortho 2014;36: 603-611.

9. Katkar RA. Cone beam CT dentistry: responsibilities of dental practitioner. Oral health case rep 2015;1: 1.

10. Shin HS, Nam KC, Park CS. Effective doses from panoramic radiography and $\mathrm{CBCT}$ (cone beam CT) using dose area product (DAP) in dentistry. Dentomaxillofac Radiol 2014;43: 5.

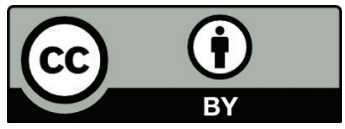

This work is licensed under a Creative Commons Attribution 